\title{
MiR-181a Promotes Spermatogenesis by Targeting the S6K1 Pathway
}

\author{
Lei Wang ${ }^{1}$, Juan Sun ${ }^{2}$, Jin $\mathrm{Han}^{1}$, Zhaowen $\mathrm{Ma}^{1}$, Meiling $\mathrm{Pan}^{1}$, Zhaojin $\mathrm{Du}^{3}$ \\ ${ }^{1}$ Reproductive Medical Center, Zaozhuang Maternal and Child Health Hospital, Zaozhuang, China \\ ${ }^{2}$ Department of Gynaecology, Zaozhuang Maternal and Child Health Hospital, Zaozhuang, China \\ ${ }^{3}$ Reproductive Medical Center, Qingdao Women and Children's Hospital, Qingdao University, Qingdao, China
}

\begin{abstract}
Approximately $15 \%$ of couples suffer from infertility worldwide, and male factors contribute to about $30 \%$ of total sterility cases. However, there is little progress in treatments due to the obscured understanding of underlying mechanisms. Recently microRNAs have emerged as a key player in the process of spermatogenesis. Expression profiling of miR-181a was carried out in murine testes and spermatocyte culture system. In vitro cellular and biochemical assays were used to examine the effect of miR-181a and identify its target $\mathrm{S} 6 \mathrm{~K} 1$, as well as elucidate the function with chemical inhibitor of S6K1. Human testis samples analysis was employed to validate the findings. miR-181a level was upregulated during mouse spermatogenesis and knockdown of miR-181a attenuated the cell proliferation and Gl/S arrest and increased the level of S6K1, which was identified as a downstream target of miR-181a. Overexpression of S6K1 also led to growth arrest of spermatocytes while inhibitor of S6K1 rescued the miR-181a knockdown-mediated cell proliferation defect. In human testis samples of azoospermia patients, low level of miR-181a was correlated with defects in the spermatogenic process. miR-181a is identified as a new regulator and high level of miR-181a contributes to spermatogenesis via targeting S6K1.
\end{abstract}

Keywords: MiR-181a, Spermatogenesis, Male infertility, S6K1

\section{Introduction}

Infertility besets about $15 \%$ of couples at childbearing age over the world, which equals about $48 \sim 72$ million couples according to the World Health Organization.

Received: January 2, 2021, Revised: February 11, 2021,

Accepted: March 17, 2021, Published online: April 30, 2021

Correspondence to Zhaojin Du

Reproductive Medical Center, Qingdao Women and Children's Hospital, Qingdao University, No. 6 Tongfu Road, Shibei, Qingdao 266034, China

Tel: +86-18553263007, Fax: +86-18553263007

E-mail: doctorduzhaojin@163.com

(a) This is an open-access article distributed under the terms of the Creative Commons Attribution Non-Commercial License (http://creativecommons.org/ licenses/by-nc/4.0/), which permits unrestricted non-commercial use, distribution, and reproduction in any medium, provided the original work is properly cited.

Copyright (c) 2021 by the Korean Society for Stem Cell Research
Female factors typically account for $50 \%$ of the cases while male factors are responsible for about $20 \sim 30 \%$ of the cases. It is estimated that $2 \sim 7 \%$ of all men exhibit different levels of sub-optimal sperm characteristics, such as alteration in sperm motility, morphology and sperm concentration $(1,2)$. There is a deteriorating trend in the last few decades as continued decrease of sperm count has been reported from different regions of the world $(3,4)$. The causes behind male infertility are complex, including the genetic mutation like Klinefelter syndrome with two $\mathrm{X}$ chromosomes and one $\mathrm{Y}$ chromosome, obstructive reproduction duct, immune disorder and inflammation, genital disorders, environmental exposure, and malfunction in the production of sperm $(5,6)$. Azoospermia is one of the severe types of male infertility, it is a medical condition that no sperm is contained in semen due to various defects in the process of spermatogenesis or ductal system of testes $(7,8)$. Of note, in the special case called ob- 
structive azoospermia, spermatogenesis is normal but no spermatozoa could be found in the ejaculate. Obstructive azoospermia is commonly seen as urologic disease for about $6.1 \sim 13.6 \%$ of total male infertility patients $(9,10)$. The treatment for male infertility is very limited and huge unmet needs exist (11-14).

Germ cells undergo a fundamental and complicated process called spermatogenesis within the seminiferous tubule epithelium. Spermatogenesis includes one cycle of DNA replication and two consecutive cell divisions, during which dramatic changes occur in the cell cycle, growth, differentiation and morphology (15). Spermatogenesis is briefly divided into three successive phases: mitosis, meiosis and spermiogenesis. Spermatogonia first replicate the whole set of genes and divide into primary spermatocytes in mitosis like normal somatic cell division. Besides the self-renewal of spermatogonia, some offspring cells detach from the basement membrane and enter meiosis. Primary spermatocytes then undergo the second cell division without DNA replication and give rise to the haploid spermatids in the stage of meiosis. Finally, the spermatids further differentiate and undergo morphogenesis into spermatozoa in spermiogenesis. The spermatozoa are fully activated in the process called capacitation and are competent to fertilize the oocyte (16). During the process there is complex interplay between germ cells and supporting somatic cells, which requires cell and time-specific expression of a large set of genes and post-transcriptional regulation of genes and their products governing the diverse biological processes, including cell cycle and proliferation, DNA replication and chromatin remodeling, energy metabolism, mobility and morphology control (17). Many fundamental pathways are involved, such as MAPK (mitogen-activated protein kinase) (18), PI3K (phosphatidylinositol-4,5-bisphosphate 3-kinase) (19), TGF- $\beta$ (transforming growth factor beta) (20) and mTOR (mammalian target of rapamycin) pathways (21). However, the detailed mechanism of gene regulation is still elusive.

Recently, microRNAs (miRNAs, miRs) have been implicated in the complex network of gene regulation in the process of spermatogenesis $(22,23)$. MiRNAs are small non-coding RNAs of 19-23 nucleotides length which can bind to the target mRNAs through Watson-Crick base pairing and eventually cause the cleavage and degradation of mRNAs through the RISC (RNA-induced silencing complex) machinery (24). There have been multiple reports on miRNA's roles in spermatogenesis, such as miR-21, let-7, miR-17-92, miR-34c and miRNA-146 (25-28). Some miRNAs are considered as potential biomarker for male infertility, for instance miR-141 is often upregulated in oligoasthenozoospermia (29). Genetic knockout studies suggest that loss of RISC-related components such as Dicer is linked with different defects of spermatogenesis (30), suggesting the importance of miRNA pathway in male germ cell development.

To shed more light on the role of miRNA in spermatogenesis, we studied the expression profile of miR-181a and established the connection between miR-181a and S6K1 in mouse spermatogenesis. miR-181a was highly expressed in testis tissues, especially the spermatogonia but not in spermatids. MiR-181a could downregulate S6K1 level and promote the cell proliferation of spermatogonia. Inhibition of miR-181a could suppress the cell proliferation which could be reversed by S6K1 inhibitor treatment. Interestingly, low level of miR-181a expression was observed in human male infertile patients with maturation arrest or hypospermatogenesis, compared to obstructive azoospermia patients which had normal spermatogenesis, suggesting the essential role of miR-181a to promote spermatogenesis.

\section{Materials and Methods}

\section{Reagent}

S6K1 inhibitor (PF-4708671, PZ0143\#) was purchased from Sigma-Aldrich (St. Louis, MI., USA). Propidium iodide/RNase solution (\#550825) was from Becton Dickinson (San Jose, CA., USA). MiR-181a mimic and inhibitor (MIH 01386) was purchased from Applied Biological Materials (ABM, Richmond, BC, Canada). RNeasy mini kit (74106\#) was from QIAGEN (Valencia, CA., USA). Antibodies for mTOR1 (2972\#), S6K1 (2708\#), pS6K1 (9204\#) were from Cell Signaling Technology (Danvers, MA., USA). GADPH (0411\#) antibody was from Santa Cruz Biotechnology (Dallas, TX, USA). MTT cell proliferation reagent (M6494\#), Lipofectamine 2000 (12566014\#), TaqMan MiRNA assay (4440886\#) were from Thermo Fisher (Waltham, MA., USA). Dual luciferase assay kit (E1910) was from Promega (Madison, MI., USA).

\section{Animals}

C57BL/6 mice were from Shanghai SLAC Laboratory Animal Co. Ltd. Animals were housed in full ventilated cages in temperature controlled $\left(20 \sim 24^{\circ} \mathrm{C}\right)$ certified SPF grade facilities. All animals have free access to food and water unless indicated otherwise. All animal protocols were approved by the Institutional Animal Care and Use Committee of Qingdao Women and Children's Hospital, Qingdao University in accordance with the guidelines of the National Institutes of Health Guide for the Care and Use of Laboratory Animals. The testis tissues were col- 
lected from male mice at different stages after birth, various tissues from adult male mice were analysed for the expression of miR-181a, after the euthanasia of animals with $\mathrm{CO}_{2}$ inhalation and cervical dislocation.

\section{Cell culture and transfection}

GC-1 spg (CRL-2053\#) and GC-2 spd (CRL-2196\#) cell lines were from American Type Culture Collection (ATCC, Rockville, MD, USA). The cells were maintained in Dulbecco's modified Eagle's medium (DMEM) supplied with $10 \%$ foetal bovine serum (FBS, Hyclone), $2 \mathrm{mM}$ L-glutamine, and 1\% antibiotics (penicillin and streptomycin) at humidified incubator with $5 \% \mathrm{CO}_{2}, 37^{\circ} \mathrm{C}$. miRNA mimic or inhibitor was transfected into cells with Lipofectamine 2000 following the instruction of manufacturer.

\section{RT-qPCR assay}

Total RNA was extracted by RNeasy kit (Qiagen) and 25 ng total RNA was used for reverse transcription reaction. miRNA quantification was performed with TaqMan miRNA assay according to the manufacturer's instruction. Nucleolar small RNA RNU44 was used as internal control.

\section{Western blotting}

Total cell lysates were extracted with RIPA buffer and equal number of lysates were resolved on SDS-PAGE followed by standard immunoblotting procedures with indicated antibodies. The data were representative of three independent experiments.

\section{MTT proliferation assay}

GC-1 or GC-2 cells were plated at 2,000 cells/well density in 96 well plate and subject to different treatment/ transfection. After $48 \mathrm{~h}$, cell medium was removed and after wash with PBS, fresh medium supplied with MTT reagent $(0.3 \mathrm{mg} / \mathrm{ml})$ was added to each well. The plate was scanned at plate reader $(570 \mathrm{~nm})$ for absorbance. Data were analysed with the Graphpad software.

\section{Luciferase reporter assay}

3'UTR of miR-181a (300 500 nt) was inserted into pMirGlo vector, mutation at seed region was introduced as designed. $48 \mathrm{~h}$ after transfection into GC-1 spg cells and various treatment, cell lysate was prepared and measured according to the instruction of manufacture (Promega dual luciferase kit). The results were analysed with Graphpad software.

\section{Cell cycle profiling}

Cells were treated with different conditions and then stained with propidium iodide (PI) solution after two brief washes with PBS and incubated at room temperature for 30 minutes with PI/RNase solution. Samples were analyzed on a FACScan flow cytometer (Becton Dickinson, San Jose, CA., USA). At least 20,000 events were collected on each sample. Cell cycle analysis of DNA histograms was performed with FlowJo software (Tree Star Inc., Ashland, OR., USA).

\section{Human testicular samples analysis}

Biopsy samples of 20 patients (aged 25 40 years) diagnosed with Non-obstructive azoospermia (NOA) were obtained with approval of IRB committee in Qingdao Women and Children's Hospital, Qingdao University. Written informed consent were collected from patients before their enrolment into this study.

\section{Statistics}

All data were presented as means \pm S.D. Data was analysed by one- or two-way ANOVA analysis followed by a post hoc test. $\mathrm{p}<0.05$ was considered as statistically significant.

\section{Results}

miR-18la was upregulated during spermatogenesis

To understand the role of miR-181a in mouse physiology, we profiled the expression level of miR-181a in different tissues of adult mice. As shown in Fig. 1A, the relative expression level of miR-181a of testis was the highest in adult male mice, followed by high levels in the kidney and thymus, compared to other tissues such as the brain, heart, spleen, muscle and skin. This indicated miR-181a was relevant to the function of testis. We next examined the time course of miR-181a expression in the different stages of postnatal mouse testes. From day 7 to 21, the miR-181a level showed a marginal increase from 100 to 130 with day 7 at relative level of 100. From day 28 to 42, the level increased dramatically to more than 300 (Fig. 1B). Maximum level was seen at more than 450 in the sample of day 42. This pattern matched the progress of spermatogenesis in mouse in which the mature spermatozoa are first produced in male mouse around 5 weeks after birth. The results demonstrated that miR-181a was enriched in male reproductive organ and upregulated in testis tissue during the spermatogenesis.

miR-181a promoted spermatogonia cell proliferation

To assess the role of miR-181a in spermatogenesis, we use the in vitro spermatogenic murine cell culture model 

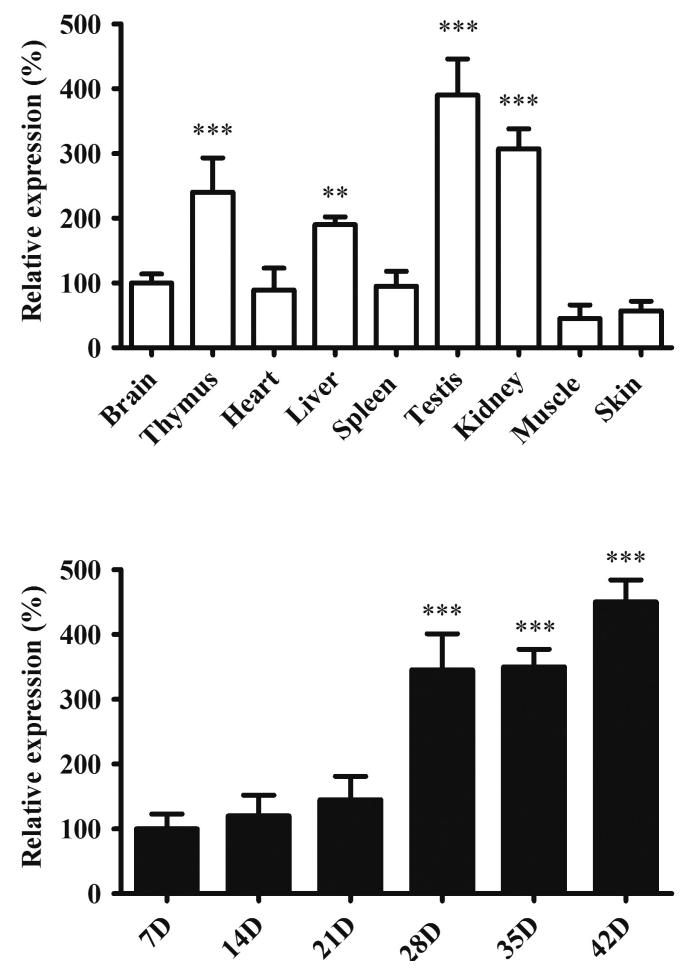

Fig. 1. miR-181a was upregulated during spermatogenesis. (A) Relative expression of miR-181a in different mouse tissues. The relative change of the miRNA in different tissues was normalized to that of the brain $\left({ }^{* * *} p<0.001\right.$ as compared with skin tissues). (B) Relative expression of miR-181a at different postnatal ages of mouse testes. The relative change of the miRNA at different time points was normalized to that of day 7 postnatally. Values were presented as mean $\pm S D(n=6, * * * p<0.001$ as compared with the sample of day 7 ).

GC-1 and GC-2 cell lines. GC-1 spg cell resembles the transition between spermatogonia and primary spermatocytes and GC-2 spd (ts) cell is spermatocyte arrested at premeiotic stage. Firstly miR-181a expression level was determined in these cells. Compared to fibroblast NIH3T3 and myoblast $\mathrm{C} 2 \mathrm{C} 12$ cell lines, GC-1 spg cells showed the highest level of miR-181a which was about 3-6 times higher than that in NIH3T3 and C2C12. MiR-181a level in GC-2 spd cells was about the $40 \%$ of that in GC-1 spg cells, as shown in Fig. 2A. Next, we knocked down the level of miR-181a by specific inhibitor in GC-1 spd cells to monitor the cell proliferation and cell cycle profile. MTT cell proliferation assay result demonstrated the significantly reduced cell growth rate in miR-181a inhibitor transfected cells compared to scramble control (Fig. 2B). There was obvious G1-S arrest in miR-181a inhibitor transfected cells compared to control (Fig. 2C), as sig-

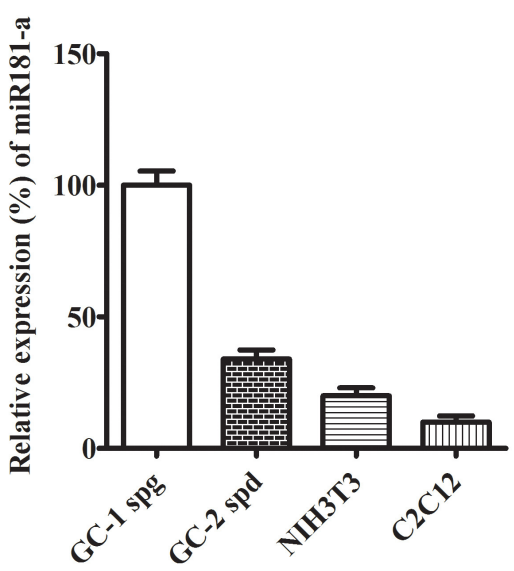

B

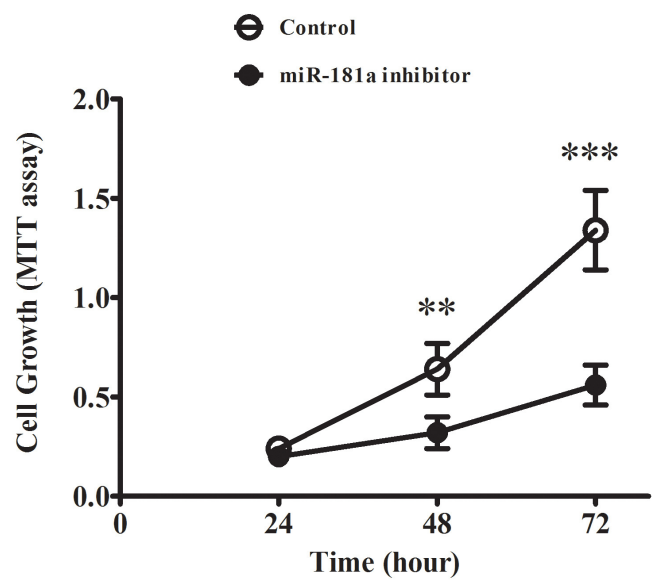

C

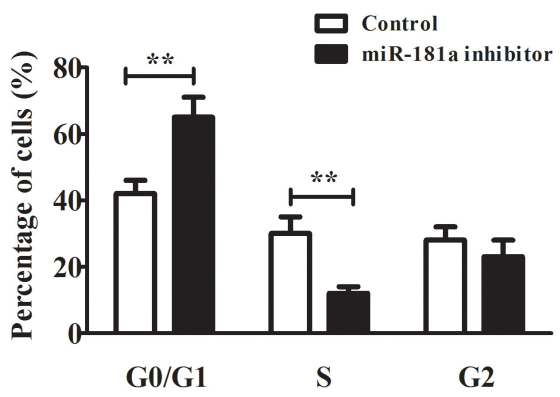

Fig. 2. miR-181a regulated GC-1 spg proliferation. (A) Relative expression of miR-181a in GC-1 spg, GC-2 spd, NIH3T3 and C2C12 cell lines. The miRNA level in GC-1 spg was set to $100 \%$. (B) Cell proliferation analysis of GC-1 spg cells transfected with either miR-181a inhibitor or scramble control. The growth rate of cells was monitored using MTT kit for $72 \mathrm{~h}$. (C) Representative histogram data of the cell-cycle analysis of GC-1 spg cells transfected with either miR-181a inhibitor or scramble control. The representative images from at least three independent experiments were shown. The data were presented as means $\pm \mathrm{SD}\left({ }^{* *} \mathrm{p}<0.01,{ }^{* * *} \mathrm{p}<\right.$ 0.001 as compared with control).

nificantly less cells at $S$ stage were detected in the cells transfected with miR-181a inhibitor. These results suggested that miR-181a was highly expressed in spermatogo- 
nia stage and was required for cell proliferation.

\section{S6K1 was downstream target of miR-18la in GC-1 cells}

To find the downstream target of miR-181a, we use bioinformatic tool to identify potential match between the 3'UTR and seed region of miR-181a. One of the hits is S6K1 kinase which is an important protein kinase in mTOR signaling. mTOR is essential for cell survival, protein synthesis and energy metabolism. It has been reported to play a key role in spermatogenesis. To validate the in-silicon finding, we designed the mutant 3'UTR region of S6K1 and inserted into a luciferase reporter construct. Mutation at seed region on 3'UTR was introduced to disrupt the recognition of miR-181a as shown in Fig. 3A. Both of the luciferase assay reporters were transfected into GC-1 spg cells first, then the mimic of miR-181a or scramble control were transfected into cells. The luciferase assay result showed that miR-181a mimic could significantly suppress the reporter activity of wildtype S6K1 3'UTR. The suppression was abolished on mutant 3'UTR reporter construct, as shown in Fig. 3B. To further validate the result of luciferase assay, we examined the endogenous S6K1 protein level when miR-181a was transfected into GC-1 spg cells. Strikingly, S6K1 and phosphorylated S6K1 protein was completely undetectable in the miR-181a mimic transfected cells compared to scramble control (Fig. 3C), consistent with luciferase assay result. Collectively, these results confirmed that $\mathrm{S} 6 \mathrm{~K} 1$ was the downstream target of miR-181a.

\section{S6K1 over-expression attenuated GC-1 spg cell proliferation}

Next, we examine the function of S6K1 in GC-1 cell culture. In accordance with previous result, $\mathrm{S} 6 \mathrm{~K} 1$ protein level was much lower in GC-1 spg cells which had higher level of miR-181a, compared to GC-2 spd cells, as shown Fig. 4A. mTOR protein level was not changed between these two cell lines, suggesting the effect of miR-181a was very specific. To further assess the role of S6K1 in cell growth and differentiation, wildtype or kinase dead mutant form of S6K1 was over-expressed in GC-1 spg cells. As shown in MTT assay (Fig. 4B), cells overexpressed with wildtype S6K1 protein showed significantly reduced cell proliferation compared to cells with GFP control or S6K1 kinase dead mutant protein. Similarly, cells with wildtype S6K1 overexpression showed remarked G1/S arrest, compared to cells with GFP control or kinase dead S6K1 overexpression (Fig. 4C). This is in line with previous result in Fig. 2C, where inhibition of miR-181a showed Gl/S arrest. These results demonstrated that S6K1 suppressed the spermatocyte proliferation in the manner dependent on its kinase activity.

\section{Inhibiting S6K1 activity rescued the antagonizing effects of miR-18la inhibitor in GC-1 spg cell growth}

To further explore the relation between miR-181a and
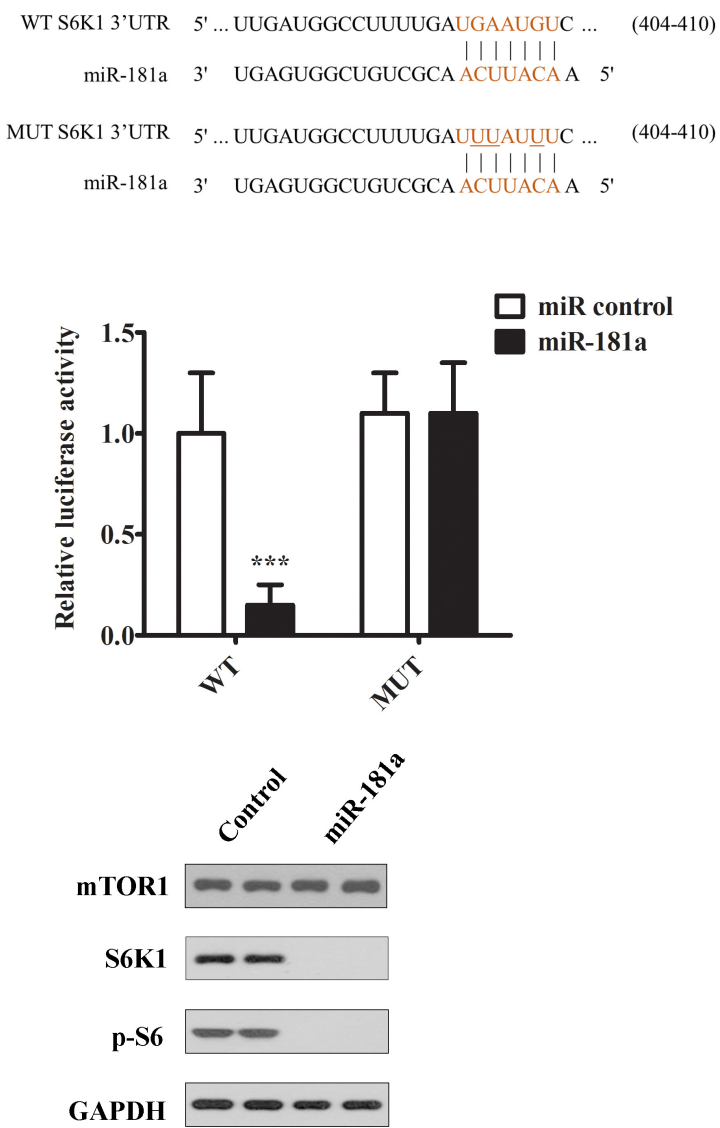

Fig. 3. S6K1 was downstream target of miR-181a in cells. (A) Schematic diagram of a conserved putative miR-181a targeting site in 3'UTR of S6K1 (WT). The site mutations were introduced to miR-181a targeting site of $3^{\prime}$ UTR (MUT). (B) Luciferase reporter assay study of the interaction between miR-181a and 3'UTR of S6K1. GC1 spg cells were transfected with luciferase reporter genes conjugated with either wildtype (WT) or mutant (MUT) 3'UTR of S6K1. 24 hours later, the cells were transfected further with either miR-181a mimic or scramble control. Transfected cells were cultured for 48 hour and then harvested for the measurement of luciferase activities in a plate reader. (C) Western blotting analysis of mTOR1, S6K1, p-S6K1 in cells transfected with either miR-181a or scramble control. The representative image from at least three independent experiments was shown. The representative images from at least three independent experiments were shown. The data were presented as means $\pm \mathrm{SD}\left({ }^{*} \mathrm{p}<0.05,{ }^{* *} \mathrm{p}<0.01,{ }^{* * *} \mathrm{p}<\right.$ 0.001 as compared with the control). 


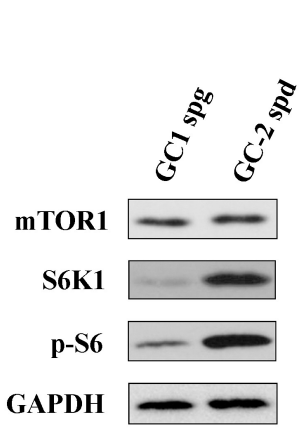

C

A

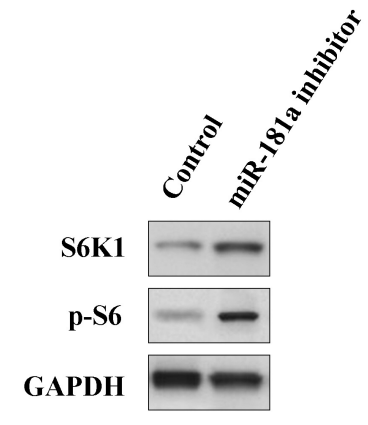

B
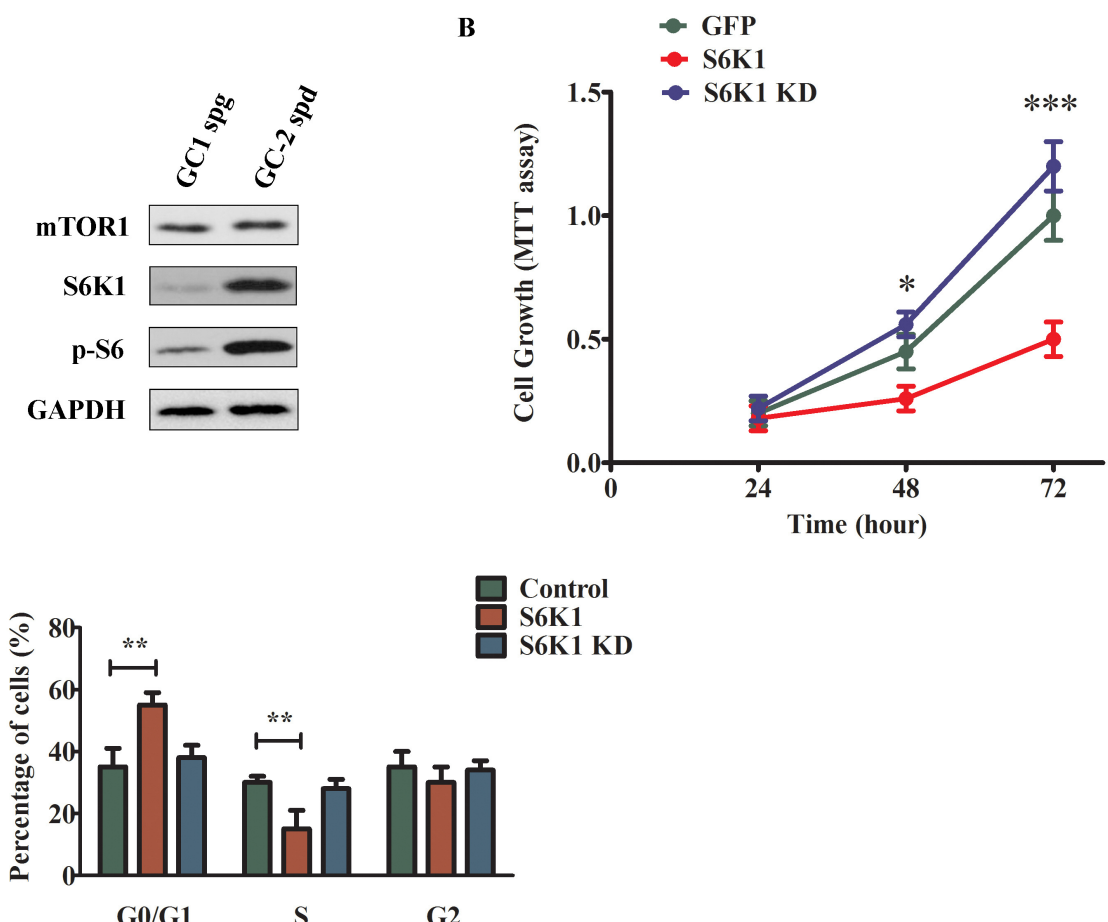

Control

S6K1

6K1 KD
G2
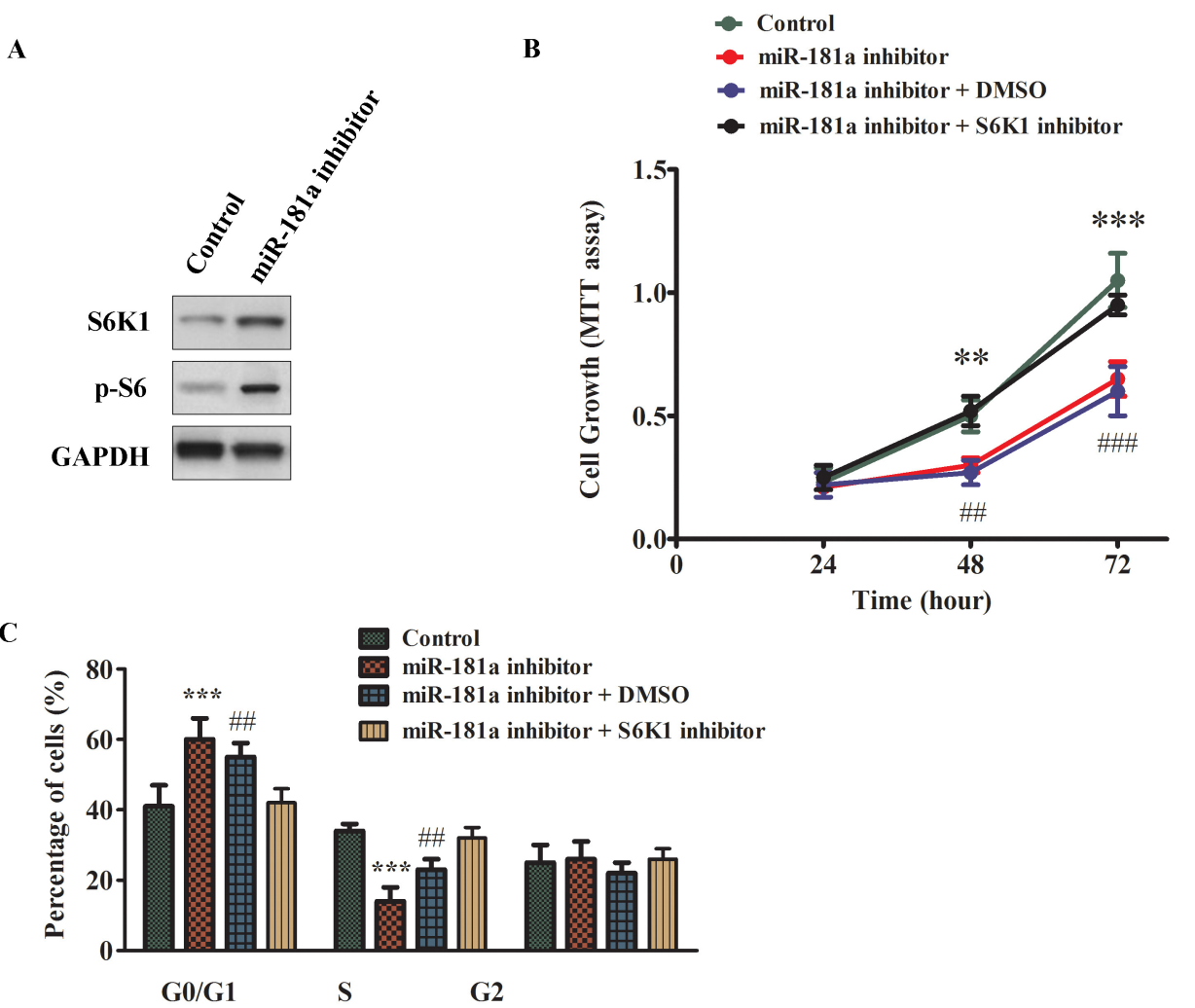

S6K1, we introduced miR-181a inhibitor into GC-1 spg cells and observed the dramatic increase of S6K1 protein level compared to scramble control as shown in Fig. 5A,
Fig. 4. S6K1 over-expression attenuated GC-1 spg cell proliferation. (A) Western blotting analysis of mTOR1, S6K1, p-S6K1 in GC1 spg and GC-2 spd cell lines. (B) Cell proliferation analysis of GC-1 spg cells transfected with GFP or S6K1 or S6K1 kinase dead mutant. The growth rate of cells was monitored using MTT kit for 72 h. (C) Representative histogram data of the cell-cycle analysis of GC-1 spg cells transfected with transfected with GFP or S6K1 or S6K1 KD mutant. The representative images from at least three independent experiments were shown. The data were presented as means \pm SD $\left(* \mathrm{p}<0.05,{ }^{* *} \mathrm{p}<0.01,{ }^{* * *} \mathrm{p}<\right.$ 0.001 as compared with control).

Fig. 5. Inhibiting S6K1 activity rescued the antagonizing effects of miR-181a inhibitor in GC-1 spg cell growth. (A) Western blotting analysis of S6K1 and p-S6K1 in GC1 spg cells transfected with control or miR-181a inhibitor. (B) Cell proliferation analysis of GC-1 spg cells transfected with control or miR-181a inhibitor. In parallel, the miR-181a inhibitor transfected cells were also treated with S6K1 kinase inhibitor or DMSO. The growth rate of cells was monitored using MTT kit for $72 \mathrm{~h}$. (C) Representative histogram data of the cell-cycle analysis of GC-1 spg cells in different treatment conditions as described above. The representative images from at least three independent experiments were shown. The data were presented as means \pm SD $\quad(* \mathrm{p}<0.05, * * \mathrm{p}<0.01$, $* * * \mathrm{p}<0.001$ as compared with control). clearly suggesting that S6K1 was directly downregulated by miR-181a. We then examined whether S6K1 kinase inhibitor could affect miR-181a function. GC-1 spg cells 


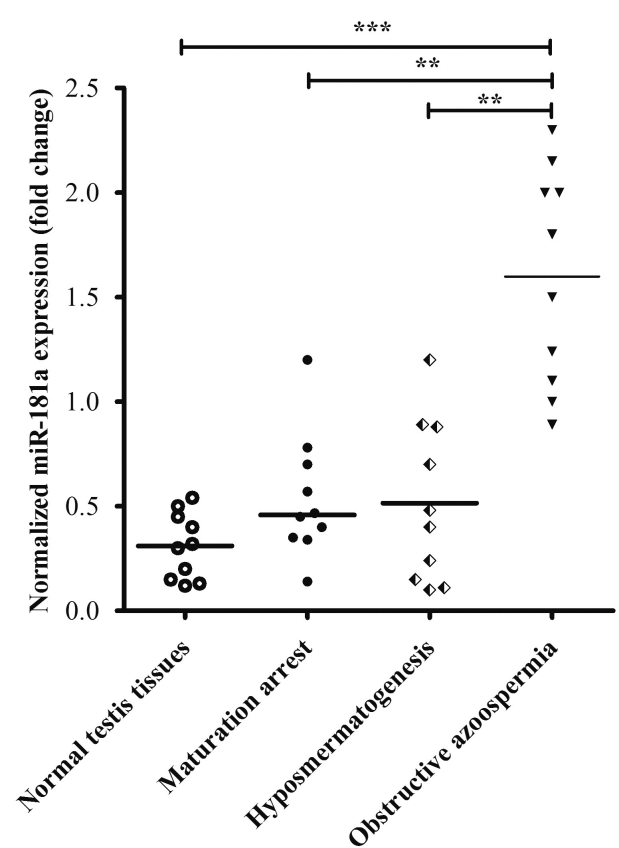

Fig. 6. miR-181a was downregulated in the testis tissues of sterile men with non-obstructive azoospermia. miR-181a expression level in testis of patients with maturation arrest, hypo-spermatogenesis or non-obstructive azoospermia was quantified by real time PCR $(\mathrm{n}=10$ for each group and $* * \mathrm{p}<0.01$ as compared with non-obstructive azoospermia patient group). The expression levels of the mRNA were also quantified in normal testis tissues from post-mortal donors.

were transfected with miR-181a inhibitor or scramble control first, then S6K1 inhibitor or DMSO control vehicle was added to the cells with miR-181a inhibitor transfection. As shown in Fig. 5B, cells with miR-181a inhibitor showed decreased proliferation compared to cells with scramble control, this suppressive effect on cell growth was reverted by $\mathrm{S} 6 \mathrm{~K} 1$ inhibitor treatment. Likewise, miR-181a inhibitor could cause significant G1/S arrest as shown by cell cycle profiling in Fig. 5C, and S6K1 inhibitor treatment could recover the cell population in $\mathrm{S}$ phase to the level of scramble control. These results indicated that S6K1 was the downstream factor of miR-181a to regulate cell growth and inhibitor of S6K1 was effective to revert the suppressive role of low level of miR-181a.

\section{miR-181a was downregulated in the testis tissues of sterile men with non-obstructive azoospermia}

To extend our finding in mouse cellular model of spermatogenesis, we assessed the level of miR-181a in human testis samples by RT-qPCR method. In non-obstructive azoospermia such as maturation arrest or hypo-spermatogenesis type of male infertility patients, the level of
miR-181a was found at relatively low level, as shown in Fig. 6. However, miR-181a level was significantly higher in the patients of obstructive azoospermia in which the spermatogenesis is normal. In addition, we also included the expression levels of miRNA in normal testis tissues as comparison and observed a slightly lower expression than the sterile patients from maturation arrest or hypo-spermatogenesis types. This result indicated that high level of miR-181a was well correlated with normal spermatogenesis, in accordance with the previous results from in vitro cell model. Future study will be performed to establish the role of S6K1 in these patients and test the therapeutic potential of S6K1 kinase inhibitor in the non-obstructive azoospermia patients with low level of miR-181a.

\section{Discussion}

Male infertility is a common cause for couples who have difficulty to bear a child. Azoospermia is a severe type of male infertility with elusive mechanisms and limited available therapy. MiRNAs have been implicated to play a role in the spermatogenesis. Here we reported a new mechanism of miR-181a in this process. MiR-181a is highly enriched in male reproductive organ and increased expression level was observed in the maturation peak of spermatogenesis. Using a murine spermatocyte culture system, we demonstrate that miR-181a is required for cell proliferation and cell cycle progression. Mechanistically miR-181a could target S6K1 protein kinase of mTOR pathway by 3'UTR reporter assay and protein immunoblotting. Further we found S6K1 overexpression in spermatocyte could suppress the cell growth and kinase activity is essential for the suppression. On the other hand, S6K1 kinase inhibitor could revert the cell growth arrest by knocking down miR-181a level. In other words, S6K1 kinase inhibitor could compensate the effect caused by low level of miR-181a. Lastly human testis sample from male infertility patients revealed the correlation of high level of miR-181a with functional spermatogenesis. Our study established the role of miR-181a-S6K1 axis in spermatogenesis and pointed out the potential therapeutic application of miR-181a mimic and S6K1 protein kinase inhibitor in non-obstructive azoospermia.

Spermatogenesis involves the highly orchestrated waves of transcriptional and translational regulation of gene networks in the different cell types like gametes and somatic cells. Notably, only a small fraction of human genome is transcribed into coding mRNAs. Recently non-coding RNAs have attracted increased attention and play important functions in multiple biological processes. Among 
them, microRNAs are small non-coding RNAs which constitute the fine-tune control of huge number of coding genes as a supplement to conventional gene regulation. Many miRNAs have been reported to play important role in spermatogenesis, for example, miR-15a (31), miR-18 (32), among others as reviewed $(22,33)$. A microarray profiling of miRNAs in mouse testes has mentioned the potential role of miR-181 family members (34). However, our study provides the thorough mechanistic insight of miR-181a in spermatogenesis and identifies the downstream target S6K1 which is the downstream key a serine/threonine kinase activated by mTOR. S6K1 is considered a master regulator of cellular metabolism and survival together with mTOR and its downstream effector ribosomal protein S6 (35-37). mTOR has been reported to be important for Sertoli cell polarity and spermatogenesis in human (38). A recent study of mTOR and S6K1 in rat spermatogenesis reported some seemingly contradictory findings with our results (21). Their conclusion that mTOR/ p70S6K1 promotes spermatogonia was based on the observation from 8-week, 38-week and 80-week old rats. The effect of decrease in mTOR signaling is marginal in their results and the significant change at 80 -week rats is more about aging instead of spermatogenesis which should be active at much earlier stage of life. In addition, our result was supported by another miRNA study in mouse spermatogenesis that activation of mTOR pathway is detrimental to cell survival (39). The study using knockout miR-17-92 offered the solid proof on its role in spermatogenesis. What's more, gene disruption of S6K1 in mice does not cause male infertility $(35,36)$, supporting our finding with S6K1 inhibitor (Fig. 5B and 5C). We think the discrepancy between our results and $\mathrm{Xu}$ et al. (21) report may be originated from different species used and different stages of sperm development under observation. The method difference is also noticeable. Xu et al. (21) observed that rapamycin treatment in 8-week male mice led to decreased sperm count which was consistent with human study with rapamycin. However, whole body administration of mTOR inhibitor rapamycin may have profound effects. It is reported that mTOR is essential for the polarity maintenance of the Sertoli nursing cells by controlling the tight gap junction in addition to regulation of cell metabolism (40). Our finding is based on the spermatocyte itself rather than focusing on the Sertoli cell and germ cell interaction. Loss of all members of miR-181 clusters leads to decreased survival, retarded growth and lymphocyte homeostasis in mice (41). This report is largely in line with our finding on cell proliferation, although there might be different functions for miR-181 in various type of tissues. Therefore, further tissue-specific knockout of miR-181a subtype in male reproductive organ is required to elucidate the mechanism of miR-181a in spermatogenesis in a more physiologically relevant condition.

Activation of $\mathrm{mTOR} / \mathrm{S} 6 \mathrm{~K} 1$ protein kinase pathway is frequently observed in multiple cancers and thus targeting mTOR in cancer is under development (42). Inhibitor of S6K1 protein kinase has been evaluated in many different cancers (43-45). Inhibition of S6K1 caused the cell growth arrest and apoptosis in the setting of cancer cell lines. However, in our study we found inhibitor of S6K1 actually promoted the spermatocyte proliferation in the background of miR-181a inhibition. We speculate there might be cell type specific effect and miR-181a should have other targets besides S6K1 gene. The effect we seen was the compound consequence of inhibiting both miR-181a and S6K1. Further study is needed to dissect the detailed regulatory loop between miR-181a and S6K1, and other potential genes involved in the unique setting of spermatogenesis.

In summary, we reported the function of miR-181a in mouse germ cell differentiation and proliferation by the profiling of miR-181a expression in different tissues and development stages of male spermatogenesis and further unraveling the novel connections between miR-181a and S6K1 protein kinase as the mechanism. The arrest of cell growth caused by knockdown of miR-181a could be rescued by S6K1 kinase inhibition. Interestingly low level of miR-181a was found in sterile male testis samples compared to the ones with normal spermatogenesis. This finding opens a new avenue for the future study to investigate the inhibition of $56 \mathrm{~K} 1$ in non-obstructive azoospermia patients with low level of miR-181a.

\section{Potential Conflict of Interest}

The authors have no conflicting financial interest.

\section{References}

1. Irvine DS. Epidemiology and aetiology of male infertility. Hum Reprod 1998;13 Suppl 1:33-44

2. Winters BR, Walsh TJ. The epidemiology of male infertility. Urol Clin North Am 2014;41:195-204

3. Agarwal A, Mulgund A, Hamada A, Chyatte MR. A unique view on male infertility around the globe. Reprod Biol Endocrinol 2015;13:37

4. Kumar N, Singh AK. Trends of male factor infertility, an important cause of infertility: a review of literature. J Hum Reprod Sci 2015;8:191-196

5. Coutton C, Fissore RA, Palermo GD, Stouffs K, Touré A. Male infertility: genetics, mechanism, and therapies. Biomed 
Res Int 2016;2016:7372362

6. Zheng W, Zou Z, Lin S, Chen X, Wang F, Li X, Dai J. Identification and functional analysis of spermatogenesisassociated gene modules in azoospermia by weighted gene coexpression network analysis. J Cell Biochem 2019;120: 3934-3944

7. Lee JY, Dada R, Sabanegh E, Carpi A, Agarwal A. Role of genetics in azoospermia. Urology 2011;77:598-601

8. Schlegel PN. Causes of azoospermia and their management. Reprod Fertil Dev 2004;16:561-572

9. Wosnitzer MS, Goldstein M. Obstructive azoospermia. Urol Clin North Am 2014;41:83-95

10. Baker K, Sabanegh E Jr. Obstructive azoospermia: reconstructive techniques and results. Clinics (Sao Paulo) 2013;68 Suppl 1:61-73

11. Jung JH, Seo JT. Empirical medical therapy in idiopathic male infertility: promise or panacea? Clin Exp Reprod Med 2014;41:108-114

12. Gulino G, Stefanucci M, Antonucci M, Racioppi M, Sacco E, Pinto F, Bassi PF. [Male infertility: non-surgical therapy]. Urologia 2014;81:148-153. Italian

13. Firlej V, Barraud-Lange V, Fouchet P. Stem cell therapy for male infertility takes a step forward. Cell Stem Cell 2012;11:585-586

14. Khourdaji I, Lee H, Smith RP. Frontiers in hormone therapy for male infertility. Transl Androl Urol 2018;7(Suppl 3): S353-S366

15. Neto FT, Bach PV, Najari BB, Li PS, Goldstein M. Spermatogenesis in humans and its affecting factors. Semin Cell Dev Biol 2016;59:10-26

16. Larson EL, Kopania EEK, Good JM. Spermatogenesis and the evolution of mammalian sex chromosomes. Trends Genet 2018;34:722-732

17. Tanaka H. [Regulation of gene expression in spermatogenesis]. Tanpakushitsu Kakusan Koso 2007;52(16 Suppl): 2116-2123. Japanese

18. Almog T, Naor Z. Mitogen activated protein kinases (MAPKs) as regulators of spermatogenesis and spermatozoa functions. Mol Cell Endocrinol 2008;282:39-44

19. Guillermet-Guibert J, Smith LB, Halet G, Whitehead MA, Pearce W, Rebourcet D, León K, Crépieux P, Nock G, Strömstedt M, Enerback M, Chelala C, Graupera M, Carroll J, Cosulich S, Saunders PT, Huhtaniemi I, Vanhaesebroeck B. Novel role for p110 $\beta$ PI 3-kinase in male fertility through regulation of androgen receptor activity in Sertoli cells. PLoS Genet 2015;11:e1005304

20. Dias VL, Rajpert-De Meyts E, McLachlan R, Loveland KL. Analysis of activin/TGFB-signaling modulators within the normal and dysfunctional adult human testis reveals evidence of altered signaling capacity in a subset of seminomas. Reproduction 2009;138:801-811

21. Xu H, Shen L, Chen X, Ding Y, He J, Zhu J, Wang Y, Liu X. mTOR/P70S6K promotes spermatogonia proliferation and spermatogenesis in Sprague Dawley rats. Reprod Biomed Online 2016;32:207-217

22. Kotaja N. MicroRNAs and spermatogenesis. Fertil Steril
2014;101:1552-1562

23. Chen X, Li X, Guo J, Zhang P, Zeng W. The roles of microRNAs in regulation of mammalian spermatogenesis. J Anim Sci Biotechnol 2017;8:35

24. Gebert LFR, MacRae IJ. Regulation of microRNA function in animals. Nat Rev Mol Cell Biol 2019;20:21-37

25. Niu Z, Goodyear SM, Rao S, Wu X, Tobias JW, Avarbock MR, Brinster RL. MicroRNA-21 regulates the self-renewal of mouse spermatogonial stem cells. Proc Natl Acad Sci U S A 2011;108:12740-12745

26. Smorag L, Zheng Y, Nolte J, Zechner U, Engel W, Pantakani DV. MicroRNA signature in various cell types of mouse spermatogenesis: evidence for stage-specifically expressed miRNA-221, -203 and $-34 \mathrm{~b}-5 \mathrm{p}$ mediated spermatogenesis regulation. Biol Cell 2012;104:677-692

27. Huszar JM, Payne CJ. MicroRNA 146 (Mir146) modulates spermatogonial differentiation by retinoic acid in mice. Biol Reprod 2013;88:15

28. Tong MH, Mitchell DA, McGowan SD, Evanoff R, Griswold MD. Two miRNA clusters, Mir-17-92 (Mircl) and Mir-106b-25 (Mirc3), are involved in the regulation of spermatogonial differentiation in mice. Biol Reprod 2012;86:72

29. Abu-Halima M, Hammadeh $M$, Schmitt J, Leidinger P, Keller A, Meese E, Backes C. Altered microRNA expression profiles of human spermatozoa in patients with different spermatogenic impairments. Fertil Steril 2013;99:12491255.e16

30. Huang CC, Yao HH. Inactivation of Dicer1 in Steroidogenic factor 1-positive cells reveals tissue-specific requirement for Dicerl in adrenal, testis, and ovary. BMC Dev Biol 2010;10:66

31. Teng Y, Wang Y, Fu J, Cheng X, Miao S, Wang L. Cyclin T2: a novel miR-15a target gene involved in early spermatogenesis. FEBS Lett 2011;585:2493-2500

32. Björk JK, Sandqvist A, Elsing AN, Kotaja N, Sistonen L. miR-18, a member of Oncomir-1, targets heat shock transcription factor 2 in spermatogenesis. Development 2010;137: 3177-3184

33. McIver SC, Roman SD, Nixon B, McLaughlin EA. miRNA and mammalian male germ cells. Hum Reprod Update 2012;18:44-59

34. Yan N, Lu Y, Sun H, Tao D, Zhang S, Liu W, Ma Y. A microarray for microRNA profiling in mouse testis tissues. Reproduction 2007;134:73-79

35. Shima H, Pende M, Chen Y, Fumagalli S, Thomas G, Kozma SC. Disruption of the p70(s6k)/p85(s6k) gene reveals a small mouse phenotype and a new functional S6 kinase. EMBO J 1998;17:6649-6659

36. Pende M, Kozma SC, Jaquet M, Oorschot V, Burcelin R, Le Marchand-Brustel Y, Klumperman J, Thorens B, Thomas G. Hypoinsulinaemia, glucose intolerance and diminished beta-cell size in S6K1-deficient mice. Nature 2000;408:994997

37. Ruvinsky I, Sharon N, Lerer T, Cohen H, Stolovich-Rain M, Nir T, Dor Y, Zisman P, Meyuhas O. Ribosomal protein S6 phosphorylation is a determinant of cell size and glu- 
cose homeostasis. Genes Dev 2005;19:2199-2211

38. Tanwar PS, Kaneko-Tarui T, Zhang L, Teixeira JM. Altered LKB1/AMPK/TSC1/TSC2/mTOR signaling causes disruption of Sertoli cell polarity and spermatogenesis. Hum Mol Genet 2012;21:4394-4405

39. Xie R, Lin X, Du T, Xu K, Shen H, Wei F, Hao W, Lin T, Lin X, Qin Y, Wang H, Chen L, Yang S, Yang J, Rong X, Yao K, Xiao D, Jia J, Sun Y. Targeted disruption of miR-17-92 impairs mouse spermatogenesis by activating mTOR signaling pathway. Medicine (Baltimore) 2016;95: e2713

40. Boyer A, Girard M, Thimmanahalli DS, Levasseur A, Céleste C, Paquet M, Duggavathi R, Boerboom D. mTOR regulates gap junction alpha-1 protein trafficking in Sertoli cells and is required for the maintenance of spermatogenesis in mice. Biol Reprod 2016;95:13

41. Henao-Mejia J, Williams A, Goff LA, Staron M, LiconaLimón P, Kaech SM, Nakayama M, Rinn JL, Flavell RA. The microRNA miR-181 is a critical cellular metabolic rheostat essential for NKT cell ontogenesis and lymphocyte development and homeostasis. Immunity 2013;38:984-997

42. Meric-Bernstam F, Gonzalez-Angulo AM. Targeting the mTOR signaling network for cancer therapy. J Clin Oncol 2009;27:2278-2287

43. Park JA, Jin HO, Lee HN, Kim JH, Park IC, Noh WC, Chang YH, Hong YJ, Kim KC, Lee JK. S6K1 inhibition enhances the apoptotic cell death of breast cancer cells in response to $\mathrm{Bcl}-2 / \mathrm{Bcl}-\mathrm{xL}$ inhibition by the downregulation of survivin. Oncol Lett 2015;10:829-834

44. Choi HN, Jin HO, Kim JH, Hong SE, Kim HA, Kim EK, Lee JK, Park IC, Noh WC. Inhibition of S6K1 enhances glucose deprivation-induced cell death via downregulation of anti-apoptotic proteins in MCF-7 breast cancer cells. Biochem Biophys Res Commun 2013;432:123-128

45. Doscas ME, Williamson AJ, Usha L, Bogachkov Y, Rao GS, Xiao F, Wang Y, Ruby C, Kaufman H, Zhou J, Williams JW, Li Y, Xu X. Inhibition of p70 S6 kinase (S6K1) activity by A77 1726 and its effect on cell proliferation and cell cycle progress. Neoplasia 2014;16:824-834 